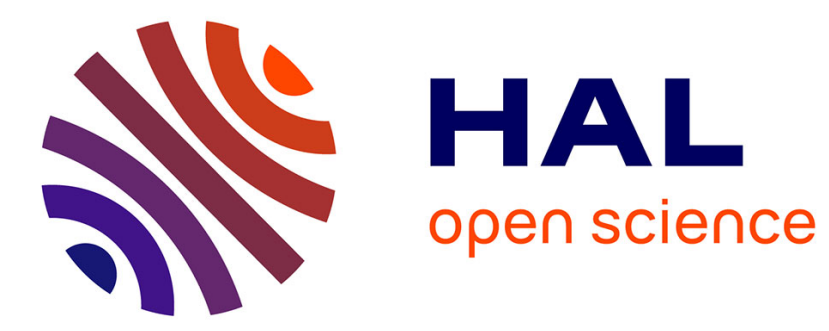

\title{
L'écriture scolaire entre stéréotype et idiolecte
}

Catherine Boré

\section{To cite this version:}

Catherine Boré. L'écriture scolaire entre stéréotype et idiolecte: variété des modes d'expression de la pensée/parole d'autrui dans les textes d'élèves. Pratiques: linguistique, littérature, didactique, 2007, 135-136, pp.217-239. halshs-00163027

\section{HAL Id: halshs-00163027 https://shs.hal.science/halshs-00163027}

Submitted on 19 Jan 2010

HAL is a multi-disciplinary open access archive for the deposit and dissemination of scientific research documents, whether they are published or not. The documents may come from teaching and research institutions in France or abroad, or from public or private research centers.
L'archive ouverte pluridisciplinaire HAL, est destinée au dépôt et à la diffusion de documents scientifiques de niveau recherche, publiés ou non, émanant des établissements d'enseignement et de recherche français ou étrangers, des laboratoires publics ou privés. 


\title{
L'écriture scolaire entre stéréotype et idiolecte
}

\author{
Catherine Boré \\ Université Cergy-Pontoise (IUFM) \\ MoDyCo UMR 7114 CNRS \\ Université Paris $X$ Nanterre
}

\section{Introduction}

Si le concept de « style » est généralement familier aux enseignants - quoique jamais explicitement défini - il n'en est pas de même de celui d'écriture. Pour Barthes, par exemple, l'écriture est « le rapport entre la création et la société, elle est le langage littéraire transformé par sa destination sociale », elle est aussi une façon de penser la «Littérature ». Ce concept était selon lui une façon d'écarter la notion de «style », trop singulière et inscrite dans l'affect de l'auteur à ses yeux pour être théorisable. Mais les nombreuses définitions ${ }^{(1)}$ qui ont suivi ont presque toutes introduit le style dans une dialectique individuel/collectif et ont souligné sa dimension sociale : somme d'idiolectes ${ }^{(2)}$, le style s'accomplit dans le discours.

Ainsi en est-il de l'écriture scolaire qu'on peut décrire comme un discours spécifique qui se constitue par l'appropriation de la langue. Elle peut être décrite comme prise entre les deux pôles du stéréotype et de l'idiolecte : c'est par exemple ce que dit Frédéric François quand il utilise le couple notionnel de communauté/différences, pour désigner ce qui, en chacun, appartient aux deux pôles du reconnaissable et de l'opaque.

C'est aussi une façon de signaler son unité comme discours commun et d'accentuer toutes les formes de sa variété ; la variété est d'évidence attestée dans l'écriture scolaire : en réponse à une consigne unique, même très contraignante, on trouve une dispersion des formes de discours produits. Mais s'il y a bien variété lexicale et morphologique, par exemple, due aux différences dans l'appropriation

(1) On peut se reporter à l'introduction de F. Neveu au colloque Styles, organisé par l'Université ParisVII-Denis Diderot, 26-27 novembre 2001, édition Sedes, qui rappelle quelques conceptions célèbres (Riffaterre, Genette, Rastier, St Gérand, etc.) on n'oubliera pas néanmoins les fondatrices: Ch. Bally et $\mathrm{M}$. Bakhtine.

(2) Parmi les nombreuses définitions concernant ce concept introduit par Bloch et développé par Hockett, je relève celle de F. Rastier dans Arts et sciences du texte (2001:179) : «...on peut appeler idiolecte l'ensemble des régularités personnelles ou " normes individuelles " dont elles témoignent [chez un émetteur]. " 
de la langue, il faut plutôt l'appeler « créativité " lorsqu'elle est transformation des structures stéréotypées de la langue.

On essaiera donc dans les pages qui suivent de clarifier les notions de stéréotype et d'idiolecte comme conditions d'une écriture scolaire, à partir des questions suivantes :

- peut-on dire que le stéréotype est l'accès à la banalité de la norme ?

- dans ce cas, serait-il le passage de l'idiolecte, éventuellement fautif, au stéréotype attendu?

- le stéréotype est-il construit (car attendu) dans la lecture (réception) des textes d'élèves?

\section{Position du problème}

\subsection{Idiolecte, style, écriture définitions barthésiennes}

Barthes a longuement réfléchi sur les pratiques enseignantes; l'enseignement du style, par exemple, est une constante obsédante de son œuvre, non résolue dans les définitions qu'il veut en donner ${ }^{(3)}$.

Surtout, Barthes dès Le degré zéro de l'écriture et tout au long de sa vie, sera revenu sur des termes voisins, idiolecte, style, écriture qu'il lie et distingue alternativement ${ }^{(4)}$. L'écriture était pour lui la forme intermédiaire entre la langue et le style, à égale distance de la contrainte du code et de la pulsion du corps ; c'était la forme idéologique du langage de l'écrivain. Les définitions de l'idiolecte et du style, quant à elles, ont toujours eu en commun l'inscription du corps dans l'élan de l'écriture. Pourtant il existe un texte très intéressant, au début de son œuvre, dans lequel Barthes, en sémioticien, propose de l'idiolecte trois définitions contradictoires :

«On retiendra pourtant que l'idiolecte peut utilement servir à désigner les réalités suivantes :

(3) Voir par exemple le $n^{\circ} 5$ de Pratiques février 1975 "Littérature et enseignement».

(4) Trois exemples parmi beaucoup d'autres :

- «Le premier objet que j'ai rencontré dans mon travail passé a été l'écriture ; mais j'entendais alors ce mot dans un sens métaphorique : c'était pour moi une variété du style littéraire, sa version en quelque sorte collective, l'ensemble des traits langagiers à travers lesquels un écrivain assume la responsabilité historique de sa forme et se rattache par son travail verbal à une certaine idéologie du langage. Aujourd'hui, 20 ans plus tard, par une sorte de remontée vers le corps, c'est au sens manuel du mot que je voudrais aller, c'est la scription sur (l'acte musculaires d'écrire, le tracé des lettres) qui m'intéresse. ("Variations sur l'écriture " Texte inédit, février 1973 dans un ouvrage collectif sur la communication pour l'Istituto accademica di Roma, texte non publié. O.C. T. IV pp. 267-268)

- "Il y a des phénomènes qu'on appelle idiolectaux; il y a un idiolecte, qui est la présence du corps. Le corps n'est peut-être pas personnel, mais il est individuel. Le corps passe d'une certaine façon dans l'écriture. Par conséquent il y a effectivement des idiolectes d'écrivain. " ( ( Où/ou va la littérature " Dialogue avec Maurice Nadeau dans I'émission « Dialogues " France-Culture 13 mars 1974, O.C. T. IV, p. 552)

- «Malgré tout, ce qu'on appelle l'écriture - c'est-à-dire le travail du corps qui est en proie au langage - passe par le style. Il y a toujours une phase stylistique dans le travail d'écriture. L'écriture commence même par le style, qui n'est pas le bien écrire : il se réfère, je le disais déjà dans Le degré zéro de l'écriture, au profond du corps, et ne peut être réduit à une intention de joliesse petitement esthétique. " (Les Nouvelles Littéraires, "Le jeu du kaléidoscope », 13 janvier 1975, Cours Entretiens, Enquêtes, O.C. T. IV pp. 846-847) 
1) le langage de l'aphasique qui ne comprend pas autrui, ne reçoit pas un message conforme à ses propres modèles verbaux, ce langage étant alors un idiolecte pur (Jakobson) ; 2) le «style » d'un écrivain, encore que le style soit toujours imprégné de certains modèles verbaux issus de la tradition, c'est-à-dire de la collectivité ; 3) on peut enfin franchement élargir la notion et définir l'idiolecte comme le langage d'une communauté linguistique, c'est-à-dire d'un groupe de personnes interprétant de la même façon tous les énoncés linguistiques ; l'idiolecte correspondrait alors à peu près à ce qu'on a tenté de décrire ailleurs sous le nom d'écriture («Le Degré zéro de l'écriture »). D'une manière générale, les tâtonnements dont témoigne le concept d'idiolecte ne font que traduire le besoin d'une entité intermédiaire entre la parole et la langue (comme le prouvait déjà la théorie de l'usage chez Hjelmslev), ou, si l'on préfère, d'une parole déjà institutionnalisée, mais non encore radicalement formalisable, comme l'est la langue. " Eléments de sémiologie 1971 « Langue et Parole » p. 92, Editions Gonthier, Pays-Bas [Le Seuil 1953 et 1964].

Entre l'idiolecte 1 qui est le « hors code » de l'aphasique, et l'idiolecte 2 qui est assimilé au style de l'écrivain, l'écart semble maximal. En outre, la réserve de Barthes à propos de l'idiolecte 2 laisse entendre que le style est toujours le produit d'un héritage, de modèles scripturaux déjà-là et l'on songe alors à l'intertextualité, qui fait de tout style une « réécriture ». Dans l'idiolecte 3, ce qui semble paradoxal, c'est que l'idiolecte désigne un langage commun à un groupe de personnes au lieu de s'appliquer à un individu singulier; mais Barthes visait ainsi le sociolecte, notion reprise à Hjelmslev. Barthes rappelle en effet que Hjelmslev a continué Saussure en opposant la langue ( «schéma » ou « forme» pour Hjelmslev) et ce qu'il appelle l'usage (ce qui correspond à peu près à " parole» chez Saussure). Mais usage et parole ne coïncident pas tout à fait: Hjelmslev assimile l'usage non à la parole de l'individu isolé, mais plus largement au langage commun de telle catégorie sociale, ce qui justifie l'appellation de « sociolecte » qui lui est attribuée.

Ces définitions vont servir de fil conducteur à cette étude. Il me semble en effet que l'écriture pratiquée à l'école emprunte quelque chose à toutes les trois.

Il existe un idiolecte 1 (négatif) de la « faute » comme du néologisme (lequel est parfois positif) : l'écriture scolaire peut alors être aussi opaque que le langage de l'aphasique, c'est le bord négatif du «style », ce qu'on pourrait appeler un style d'erreurs scolaires.

L'idiolecte 2 est l'objet même de cette étude : existe-t-il un style d'écriture scolaire? Ce serait le bord positif, qui contraindrait à rechercher des textes marqués par la créativité, c'est-à-dire la variation par rapport au modèle transmis ; les textes se démarqueraient de la norme d'écriture transmise/imposée à l'école.

Reste l'idiolecte 3, le style scolaire comme sociolecte : il serait le produit des stéréotypes de la langue apprise, acquise en commun.

Dans le parcours à venir, $\mathrm{j}$ ' approcherai la notion de style scolaire par différenciation et approximation, en gardant pour fil conducteur la série d'oppositions :

- collectif/ individuel

- communauté/différences

- reprise/variation

- normé/non normé

Je garde aussi présent à l'esprit le fait que stéréotypie, variation, norme sont affectées de valeurs variables selon la perspective envisagée. 


\subsection{Enonciation, écriture}

Dans un article très suggestif, Mathieu Valette ${ }^{(5)}$ rappelle l'historique de la distinction entre énonciation et actualisation, qui différencie l'acte d'appropriation de l'énoncé (énonciation) et la mise en acte de ce qui n'est que virtuel (actualisation). Dans la première partie de son article, à laquelle je me réfère seulement ici, il constate la « déviation » opérée par Benveniste entre l'acte individuel de parole qui chez Saussure, était l'autre face du concept de langue, alors que l'appropriation de la langue se trouve associée chez Benveniste au concept de sujet d'énonciation, avec sa problématique : la prééminence des traces laissées par le sujet de l'énonciation dans l'énoncé et des marques déictiques en général. C'est ainsi qu'avec l'énonciation, l'accent est moins mis sur le principe d'activation de la langue comme processus, que sur l'accès d'un sujet (et de ses marques) à la langue.

Cette ambiguïté dans ce que nous appelons communément «énonciation » me semble au cœur de la problématique de l'écriture scolaire, et c'est ainsi que j'aborderai la notion de "style» dans l'écriture scolaire.

En effet, dans cette acception d' « écriture » (au sens de « production d'écrit »), le style scolaire paraît n'être rien d'autre que la " mise en œuvre de la langue écrite ». L'élève scripteur effectue l'acte de s'approprier la langue, et c'est en cela que consiste l'événement énonciatif, dans son unicité et son imprévisibilité.

Avec quelque précaution, je dirais qu'on en trouverait un équivalent dans la " parole " ${ }^{(6)}$ saussurienne (ou le « discours », dans ce sens) qui passe de la potentialité à l'effectuation, et qui est bien, en effet, individuelle : l'élève scripteur est le sujet concret de son accession à la langue, comme tout sujet parlant, et c'est bien cette accession de facto à la langue qui fait de lui un sujet parlant.

Cette mise en acte de la langue par l'écriture se distingue, comme cela a été dit plus haut, de l'idée d'un scripteur «sujet d'énonciation », inscrit dans son énoncé par l'expression de son énonciation et dont il s'agirait de retrouver des marques de subjectivité. Les faits d'écriture scolaire qu'on envisage pour parler de « style » scolaire, passent par la mise en œuvre du code, et c'est l'usage qu'en fait le scripteur qui m'intéressera d'abord.

Du coup, le terme "d'appropriation » de la langue doit aussi être dégagé des deux possibilités de sens qu'il contient : l'« appropriation » de la langue par le scripteur relève d'un processus d' « acquisition » de celle-ci ; et en même temps il désigne un processus d'individuation, qui est la langue propre du scripteur. Dans le premier cas, l'accent est mis sur le "commun » dans la langue, l'appropriation du code, voire le stéréotype ; dans le second cas, sur le particulier, sur l'idiolecte.

Or ces deux sens d' " appropriation » ne se distinguent pas aussi facilement dans les écrits des jeunes scripteurs : qu'est-ce que le «commun » dans la langue ? et sous quelle forme particulière pourrait se marquer l'appropriation de la langue pour un scripteur débutant?

(5) Valette, M. (2004).

(6) Il est paradoxal d'utiliser le concept de «parole » qui chez Saussure vise les faits d'oralité, pour évoquer le style, phénomène qui vise surtout l'écrit (la «lettre » pour Saussure). Mais la transposition peut s'admettre si l'on retient dans le concept de " parole " son caractère à la fois effectif, individuel et divers, qui caractérise aussi la production écrite. Sur la question terminologique, l'articulation des concepts langue et parole dans le Cours de Linguistique Générale et surtout dans le manuscrit « De l'essence double du langage ", in Ecrits de linguistique générale (2002), je renvoie, entre autres, aux passionnants et récents articles de R. Kyheng (2005). 
Ecrire, pour ces jeunes scripteurs, révèle la langue dans son organisation et sa structure, par son envers, c'est-à-dire ses dysfonctionnements. De ce point de vue, l'individualité du scripteur se trouve ramenée à une communauté de « régularités », observables chez d'autres scripteurs de son âge, certaines généralement désignées comme des «fautes ».

Ces erreurs ont pu être systématisées comme l'avait fait H. Frei avec sa «grammaire des fautes $»$. Voici ce que dit Frei des fautes concernant par exemple la morphologie des verbes. Après avoir énoncé quatre règles majeures constitutives de cette « grammaire », il invoque la règle d'invariabilité pour expliquer ces erreurs:

«Il n'est pas exagéré de prétendre que la grande majorité des fautes de conjugaison est dictée par le besoin d'unifier le radical du verbe ; il faut que ce dernier reste inchangé en dépit de toutes les déterminations de personne, de nombre de temps et de mode qui m'atteignent. Cela revient à dire que le français tend à ramener tous ses verbes à la première conjugaison. " ${ }^{(7)}$

Cette grammaire conçue en fonction de la régularité des erreurs plutôt qu'en fonction de la résolution de celles-ci, rejoint une conception de l'idiolecte, vu en négatif (au sens du « négatif » d'une photo) comme une irrégularité partagée.

Dans les exemples ci-dessous, tirés de corpus différents, on retrouve ce qui constitue une grammaire des fautes selon Frei, c'est-à-dire la logique de ces erreurs, leur usage commun permettant d'attribuer à coup sûr à la catégorie des apprenants en général, et non pas à tel ou tel élève, l'acquisition de la morphologie.

On y remarque des erreurs morphologiques dans le paradigme du passé simple ainsi que de classiques erreurs d'accord qui pourraient s'expliquer par la règle d'invariabilité :

\section{EX1}

\section{JEN6MJ06 (Version 1)}

Un chasseur qui passait par la forêt,

et vut Soleil [c'est le nom d'un personnage]

[...]

et conclua que la boule

pouvait donner des couleurs au ciel.

[...]

Alors Soleil rentra et prena la boule...

EX2

2NIC(1)CJO7 (Version 2)

\% ...Le Cyclope aveuglé

ne me vu pas partire

EX3

10STEFI6DB04 (Version 3)

Le maire demanda maintenant

à hanté si elle voulait l'épousé elle disa

« oui ».

EX4

21THO6DB04 (Version 1)

ils se tena la main jusqu'a chez eux.

(7) Frei, H. ([1929], 1993:167). 
Des séries régulières de suites morphologiques qui n'appartiennent pas au code peuvent-elles constituer un style collectif d'erreurs?

\section{Grammaire des fautes : une stylistique des erreurs ?}

On connaît le jugement de Jakobson sur l'idiolecte qui, pour lui, ne peut qu'être une « fiction », en vertu du caractère obligatoirement social de la communication ${ }^{(8)}$, fondée sur l'adaptation et l'échange d'un code commun. Par la suite cependant, Jakobson re-examine son argument ${ }^{(9)}$ en n'admettant l'idiolecte que dans le cas des « aphasiques » qui ne comprennent que leur propre langage. L'argumentation repose sur une conception communicationnelle et dialogale de la langue, ce qui met l'idiolecte hors code, non pas en raison du code, mais en raison de l'émetteur/récepteur lui-même.

Les « fautes » communes aux élèves semblent présenter les caractères inverses : contrairement à ce qui se passe pour les aphasiques, elles sont aisément compréhensibles par les scripteurs eux-mêmes et par leurs récepteurs, même si ces derniers doivent faire un effort de réception plus important. La communication, dans ce genre de «fautes " n'est pas interrompue. En revanche, le code lui-même est quelque peu affecté dans la forme de l'expression.

La grammaire des fautes comme somme d'idiolectes classifiables représente bien un paradoxe extrême qui se dissout dès que la norme est intégrée.

En revanche, certains fonctionnements du code, non maîtrisés par les jeunes scripteurs, peuvent poser de nombreux problèmes de réception au lecteur/récepteur de productions scolaires : c'est le cas des pronoms personnels embrayeurs, que Jakobson a particulièrement étudiés et dont il a remarqué les difficultés d'acquisition chez les enfants ${ }^{(10)}$.

(8) «C'est, comme d'habitude avec un grand intérêt que j'ai lu l'article sur l'idiolecte, distribué par mon vicil ami Hockett [paru en 1958: A course in modern linguistics, chap. 28, NDA]. Cet article ramène l'idiolecte aux habitudes caractérisant le parler d'un individu particulier à un moment donné, et en exclut tout ce qui, dans les habitudes linguistiques de cet individu, se réfère à la compréhension du discours des autres. Or, si tous les propos que je tiens à Cambridge étaient observés et enregistrés sur une longue période, on ne m'entendrait jamais prononcer le mot “idiolecte". Et cependant maintenant, comme je m'adresse à vous, je l'emploie, parce que je m'adapte au langage de mes adversaires potentiels, Hockett par exemple. Et j'emploie beaucoup d'autres mots encore de la même manière. En parlant à un nouvel interlocuteur, chacun essaye toujours délibérément ou involontairement, de se découvrir un vocabulaire commun : soit pour plaire, soit simplement pour se faire comprendre, soit enfin pour se débarrasser de lui, on emploie les termes du destinataire. La propriété privée dans le domaine du langage, ça n'existe pas : tout est socialisé. L'échange verbal, comme toute forme de relation humaine, requiert au moins deux interlocuteurs; l'idiolecte n'est donc en fin de compte, qu'une fiction, quelque peu perverse. " (Jakobson, R. 1963, Tome I: 32-33)

(9) Ibid. p. 55 .

(10) «Si nous observons que même les linguistes ont eu des difficultés à définir la signification générale du terme "je" (ou "tu"), qui signifie le même fonction intermittente de différents sujets, il est tout à fait compréhensible qu'un enfant qui a appris à s'identifier à son nom propre ne s'habitue pas aisément à des termes aussi aliénables que les pronoms personnels : il peut hésiter à parler de lui-même à la première personne alors que ses interlocuteurs l'appellent " $t u$ ". Parfois il s'efforce de redistribuer ces appellations. Par exemple, il essaiera de monopoliser le pronom de première personne: "Essaie pour voir de t'appeler moi. Moi seul je suis moi et tu n'es que toi." Ou bien il usera sans discrimination soit de "je" ("moi"), soit de "tu" ("toi"), pour désigner aussi bien le destinateur que le destinataire, de sorte que le pronom désigne n'importe quel protagoniste du dialogue. " Jakobson, R. (1963) : chapitre IX, "Les embrayeurs, les catégories verbales et le verbe russe " : 180. 
Mais la difficulté de maniement des pronoms embrayeurs de première et deuxième personne décrite par Jakobson, déjà grande à l'oral, est redoublée lors du passage à l'écrit.

Les corpus de productions écrites scolaires témoignent abondamment de la difficulté du scripteur débutant à s'énoncer et à représenter l'altérité sous sa toute première forme de différenciation entre « je » et « tu ».

L'observation de ces dysfonctionnements me semble de première importance.

En effet, la lutte engagée par les scripteurs pour « instancier les signes vides de la langue " ${ }^{(11)}$ que sont les embrayeurs relève exactement de l'énonciation décrite par Benveniste :

«C'est dans l'instance de discours où « je » désigne le locuteur que celui-ci s'ćnonce comme « sujet ». Il est donc vrai à la lettre que le fondement de la subjectivité est dans l'exercice de la langue. Si l'on veut bien y réfléchir, on verra qu'il n'y a pas d'autre témoignage objectif de l'identité du sujet que celui qu'il donne ainsi lui-même sur lui-même.

Le langage est ainsi organisé qu'il permet à chaque locuteur de s'approprier la langue entière en se désignant comme je. " ${ }^{(12)}$

$\mathrm{J}$ 'ai dit plus haut que c'est dans cette accentuation particulière que Benveniste reprend Saussure, et s'en distingue en élaborant la notion de « discours ».

L'« élève dans la langue » aux prises avec ces signes marque sa manière de confronter au langage identité et altérité. Les façons d'entrer dans la langue en relation avec « tu " sont marquées de difficultés à l'écrit, plus encore qu'à l'oral.

Je donne ci-dessous quelques exemples de la diversité des postures énonciatives de « je».

\subsection{L'appropriation du système énonciatif}

\subsubsection{Exemples CE2/CM1/CM2 : instabilité énonciative des récits en « je »}

Selon les productions écrites requises par la consigne, le signe « je » peut référer soit à un personnage fictif qui dit «je», soit à un «je» de l'élève plus ou moins «fictionnalisé » en ce sens qu'il représente le scripteur en tant que sujet scolaire. Ces deux cas correspondent à une distinction grossière.

L'instabilité énonciative se produit lorsque « je » ne réfère pas à l'énonciateur en tant que personne du monde : pour les récits en « je » dont le référent coïncide avec celui de l'élève scripteur-énonciateur, le problème ne se pose pas, du moins tant que ce dernier n'est pas amené à représenter les paroles d'un autre « je », réel ou fictif.

Les exemples 5 à $8^{(13)}$ répondaient à la consigne d'écriture suivante donnée à une classe de CE2 /CM1:

«Ecrire la suite du récit surl'île des Cyclopes en utilisant la lère personne (Je ou nous). Tu es Ulysse. Ce récit doit être au présent. La dernière phrase de

(11) J'emprunte l'expression, particulièrement heureuse, à S. de Vogüe (1997:151).

(12) Benveniste, E. (1966, I : 262).

(13) Les copies sont transcrites selon l'orthographe et la présentation originales ; les ratures et autres opérations (ajouts, notamment, entre chevrons) sont également reproduites à l'identique pour chaque version, selon la transcription diplomatique. Quand c'est nécessaire (exemples 5 à 7 par exemple) une version réorthographiée est prođuite en regard. 
ton récit sera: "Nous reprenons la mer, contents d'avoir évité la mort ». Minimum : 15 lignes, maximum, 25 lignes. "

\section{EX5}

\begin{tabular}{|c|c|}
\hline $\begin{array}{l}\text { 9NAS } \\
\text { (versio }\end{array}$ & $\begin{array}{l}\text { 9NAS(1)CJ07 } \\
\text { (version } 1 \text { réorthographiée ) }\end{array}$ \\
\hline 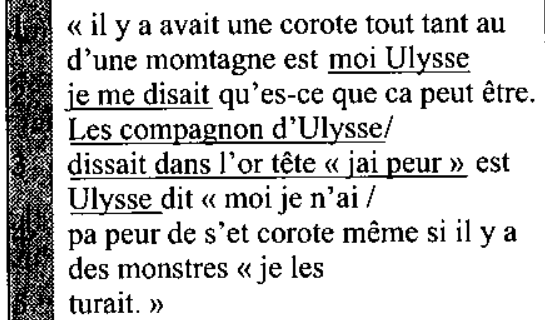 & $\begin{array}{l}\text { « il y avait une grotte tout en-haut } \\
\text { d'une montagne et moi Ulysse } \\
\text { je me disais qu'est-ce que ça peut } \\
\text { être . Les compagnons d'Ulysse/ } \\
\text { disaient dans leur tête " j'ai peur » et } \\
\text { Ulysse dit «moi je n'ai / } \\
\text { pas peur de cette grotte et même s'il } \\
\text { y a des monstres « je les } \\
\text { tuerai. » }\end{array}$ \\
\hline
\end{tabular}

Dans l'exemple 5, Ulysse parle à la première personne, comme le prescrit la consigne (1. 1-2: « et moi Ulysse je me disais... ») ; la narration s'effectue donc au moyen de « je ». Ulysse est aussi locuteur mais dans la première phrase, ses pensées ne sont pas représentées au moyen de « je » (1. 1-2: « et moi Ulysse je me disais qu'est-ce que ca peut être). Dans la phrase suivante, le scripteur introduit de nouveaux personnages désignés ainsi 1.2 : «Les compagnons d'Ulysse disaient», ce qui entraîne ipso facto un nouveau narrateur qui se substitue à Ulysse. Les pensées de ces personnages sont représentées au discours direct par « je » (1.3: «j'ai peur »). L'indice « je » devient dès lors indexé à la pensée de ces locuteurs.

Après cette phrase, il devient impossible pour le scripteur de « décrocher » de la strate énonciative qu'il a créée et de revenir à la narration en « je ». L'indice « je », quand il est repris, se réfère à l'Ulysse locuteur, et non pas narrateur (1. 3-4 "Ulysse dit " moi je n'ai pas peur...".)

Dans la version 3 (exemple 6 ci-après), le problème semble avoir disparu, à la suite d'une correction explicite de l'enseignante sur la copie, mais il reparaît ligne 9, " coupa le bras d'Ulysse " avec un retour (lignes 10-11) à la narration en « je », sans garantie de maintien du référent.

\section{EX6}

\begin{tabular}{|c|c|}
\hline $\begin{array}{l}\text { 9NAS } \\
\text { (versio }\end{array}$ & $\begin{array}{l}\text { 9NAS(1)CJ07 } \\
\text { (version } 3 \text { réorthographiée ) }\end{array}$ \\
\hline $\begin{array}{l}\text {...et je voix un cyclope } \\
\text { tout dans disant je turai Ulysse ? » Et } \\
\text { "moi je me suis [dit] « mes } \\
\text { compagnons étai partis mes moi je } \\
\text { turai ce cyclope » puis } \\
\text { un cyclope avai une ache et coupa le } \\
\text { bras d'Ylysse [sic] mes } \\
\text { moi je pris mon épée et je coupa la } \\
\begin{array}{l}\text { ganbe des deux } \\
\text { cyclope [...] }\end{array}\end{array}$ & $\begin{array}{l}\text {...et je vois un Cyclope } \\
\text { tout en disant « je tuerai Ulysse » Et } \\
\text { « moi je me suis [dit] « mes } \\
\text { compagnons étaient partis mais moi } \\
\text { je tuerai ce Cyclope » puis } \\
\text { un Cyclope avait une hache et coupa } \\
\text { le bras d'Ulysse mais } \\
\text { moi je pris mon épée et je coupai la } \\
\text { jambe des deux } \\
\text { Cyclopes [...] }\end{array}$ \\
\hline
\end{tabular}

Ce cas est loin d'être isolé. On le retrouve dans les copies d'élèves du même corpus qui ont plus d'aisance dans la narration. Dans l'exemple 7, c'est encore le changement de plans énonciatifs - passage dialogué succédant au discours narratif à la première personne puis retour à la narration - qui perturbe l'utilisation du pronom embrayeur (lignes 12-13). 
On remarque pourtant l'utilisation d'une incise de narration de discours rapporté, interne au dialogue (1. 7 « oui dis-je ») dans laquelle le changement de strate narrative et énonciative n'affecte pas l'embrayeur.

\section{EX7}

\begin{tabular}{|c|c|}
\hline & e) \\
\hline $\begin{array}{l}\text { je mavança prudament pour entré } \\
\text { dans la grote quand } \\
\text { jantandi un hurlement térible puis je } \\
\text { marêta } 1 \text { minute plus tard, je rantri } \\
\text { dans la grote il y avais un géant assis } \\
\text { qui manger une } \\
\text { bête tout autour d'un feu ; je pris mon } \\
\text { n'épé pour } \\
\text { le tué je mavança puis je marja sur un } \\
\text { n'os, le géant } \\
\text { se retourna je courus or de la grote } \\
\text { mais le géant } \\
\text { me ratrapa et me dit tu vouler me tué } \\
\text { oui dije } \\
\text { et ba puisque tu veux me tué feson un } \\
\text { comba l'oyale } \\
\text { tu peut prendre une arme moi gent né } \\
\text { pas } \\
\text { beseio besion besoin j'utilisré mais } \\
\text { fors et le } \\
\text { conba commençi le géant ésaya de } \\
\text { écrasé } \\
\text { Ulysse le généreux mais Ulysse } \\
\text { équiva les ataque } \\
\text { du monstre[... }\end{array}$ & $\begin{array}{l}\text { je m'avançai prudemment pour entrer } \\
\text { dans la grotte quand } \\
\text { j'entendis un hurlement terrible puis } \\
\text { je m'arrêtai ; une minute plus tard, je } \\
\text { rentrai } \\
\text { dans la grotte il y avait un géant assis } \\
\text { qui mangeait une } \\
\text { bête tout autour d'un feu ; je pris mon } \\
\text { épée pour } \\
\text { le tuer je m'avançai puis je marchai } \\
\text { sur un os, le géant } \\
\text { se retourna je courus hors de la grotte } \\
\text { mais le géant } 7 \text { me rattrapa et me dit } \\
\text { tu voulais me tuer oui dis-je } \\
\text { eh bien puisque tu veux me tuer fai- } \\
\text { sons un combat loyal } \\
\text { tu peux prendre une arme moi j'en ai } \\
\text { pas } \\
\text { besoin j'utiliserai mes forces et le } \\
\text { combat commença le géant essaya } \\
\text { d'écraser } \\
\text { Ulysse le généreux mais Ulysse es- } \\
\text { quiva les attaques } \\
\text { du monstre[...] }\end{array}$ \\
\hline
\end{tabular}

La même élève réussit très bien la transposition dans sa version 3. On remarque particulièrement son habileté (exemple 8 lignes 12-13) à restaurer Ulysse narrateur en première personne (" mais moi Ulysse le rusé j'esquive les attaques du monstre "), alors que par ailleurs subsistent des marques toujours incertaines d'attribution des locuteurs dans le dialogue (lignes 8-11):

\section{EX8}

\begin{tabular}{|c|}
\hline 13CIN(1) CJ07 (version 3) \\
\hline $\begin{array}{l}\text { Je m'avance prudemment pour entrer dans la grotte quand } \\
\text { j'entend un hurlement terrible. Je m'arrête. Quelques instants } \\
\text { plus tard, je rentre dans la grote il y a un géant assis } \\
\text { autour d'un feu qui mange une bête. J'ai Je prends mon } \\
\text { épée pour le tuer. Je mant m'avance prudemment mais } \\
\text { je marche [sur] un os. Le géant se retourne. Je coure hors } \\
\text { de la grote mais le géant me rattrape et me dit : « tu } \\
\text { puisque tu veux me tuer, faisons un combat loyal } \\
\text { Tu peux prendre une arme moi je en en ai pas besoin } \\
\text { j'utiliseré mes forces naturelle. Et le combat } \\
\text { commence. Le géant éssaie de m'écraser mais moi Ulysse le } \\
\text { rusé j'esquive les attaques du monstre [...] }\end{array}$ \\
\hline
\end{tabular}




\subsubsection{Incise et discours rapportés}

Dans EX9, on retrouve le problème de la confusion des plans d'énonciation évoqué plus haut : le scripteur de l'exemple 9 ne conçoit pas que « je " narrateur (=Ulysse) se maintienne comme narrateur jusque dans l'incise, alors que ce même narrateur (= Ulysse) est devenu entre- temps locuteur dans le dialogue avec le $\mathrm{Cy}$ clope, ce qui entraîne un changement de plan et de strate énonciative.

\begin{tabular}{|c|c|}
\hline $\begin{array}{l}4 \mathrm{ILY}(2) \mathrm{CJ} 07 \\
\text { Version } 1\end{array}$ & $\begin{array}{l}4 \text { ILY(2)CJ07 } \\
\text { Version } 1 \text { réorthographiée }\end{array}$ \\
\hline $\begin{array}{l}\text { Pandant le matin je suis allé dans un } \\
\text { grotte profond } \\
\text { Et je faisai un plan je vouller leur } \\
\text { faire un pieges } \\
\text { On fait un défit luit repondi Ilysse et } \\
\text { le Cyclope lui dit d'accord }\end{array}$ & $\begin{array}{l}\text { Pendant le matin je suis allé dans une } \\
\text { grotte profonde } \\
\text { Et je faisais un plan je voulais leur } \\
\text { faire un piège } \\
\text { On fait un défit lui répondit Ulysse et } \\
\text { le Cyclope lui dit d'accord }\end{array}$ \\
\hline
\end{tabular}

Ces points sont illustrés dans d'autres corpus, quelle que soit la configuration du système énonciatif, comme ci-dessous, où il s'agit de transformer une histoire fictive narrée à la troisième personne, en récit à la première personne ${ }^{(14)}$.

Dans les exemples 10 et 11 , extraits d'un corpus d'élèves de $\mathrm{CM} 2$, le récit en « je » souffre de la même instabilité que dans les exemples du corpus précédent : la narration passe de « je » à « il » en quelques lignes, mais sans que l'on puisse attribuer ce changement à un passage brutal de plans d'énonciation.

\section{EX10}

\begin{tabular}{|l|}
\hline 1ABDM2LP05 (version 1) \\
Tous les soirs mes parents rentrent du travail (mon père \\
<Dabord> \\
nommé Elie et ma mère nommée Margot) ainsi que \\
moi nommé Pedro. Tous les soirs nous écoutions la radio \\
pour s'attendre à quelque chose de grave. « Nous allons \\
dînner, à table » dit ma mère. On dîne et tout à \\
coup une méchante mauvaise [nouvelle] sort de cette \\
radio. Le père avait très peur pour sont \\
fils Pedro ainsi <que >pour Margot et pour \\
lui.
\end{tabular}

\section{EX11}

16SAB M2 LP05 (version 1)

Tous les soirs mes parents écoutent la radio au dîner. Pedro se pose toujours la même question (pourquoi il écoute la radio). Puis quand mes parents vont se coucher, ils emène prends leurs radios. A $11 \mathrm{~h}$, Pedro demande à son père Pourquoi vous prenez la radio dans votre chambre? ils n'ont pas répondue à Pedro.

(14) Les élèves ont travaillé en lecture sur des autobiographies, réelles ou fictives, et l'enjeu consiste à l'issue de ce travail de lecture à leur faire réécrire un album ( « La rédaction » éd. Syros), dont la narration est classiquement à la troisième personne, en le transformant en autobiographie rédigée par le jeune garçon héros de l'histoire, Pedro. Dans l'histoire, Pe- 


\subsubsection{Embrayeurs et discours rapportés}

Mais cette même élève qui a du mal à maintenir la cohérence du système énonciatif dans le cas d'une histoire fictive retrouve ces difficultés dans un récit pré-

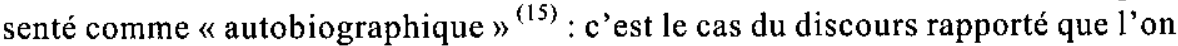
trouve ligne 16 , exemple 12 :

\section{EX12}

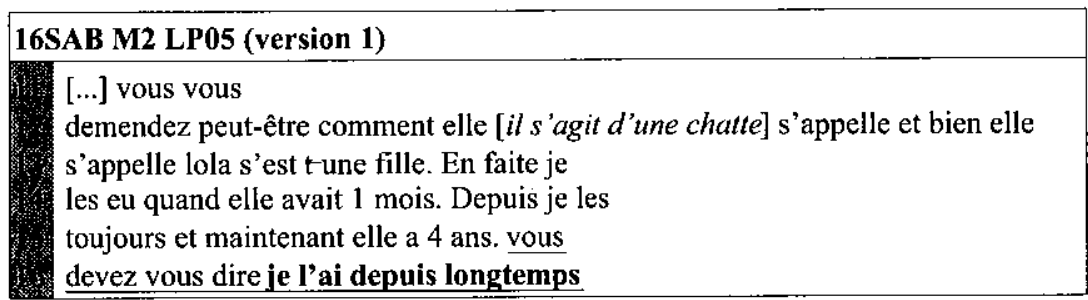

Dans cet exemple, le scripteur reproduit les pensées-paroles de l = énonciateur du message $m$ ( 1.16 « je l'ai depuis longtemps »), comme si elles étaient énoncées par L, narrateur en première personne. Or " je » ne réfère pas à l'allocutaire auquel L s'adresse sous la forme « vous » (1. 15-16: " vous devez vous dire »), alors que tout porte à le lire ainsi, mais à $L$, énonciateur-narrateur.

En fait, c'est le discours indirect que l'on attendrait ; il aurait l'avantage de conserver les marques énonciatives de L ("Vous devez vous dire que je l'ai depuis longtemps "). Mais le scripteur ne dispose pas, semble-t-il, du relateur que, qui laisserait le texte dans l'orbite de la narration en « je », et ainsi les plans sont confondus.

Cette difficulté se retrouve encore dans l'exemple suivant où, à l'inverse de l'exemple 12, la forme interrogative directe entraîne la transformation indue de l'embrayeur attendu « tu », en pronom de troisième personne. Là encore, le discours indirect ne peut pas être construit, faute du relateur adéquat ( $s i$ interrogatif)

\section{EX13}

10LAW M2 LP05 (Version 1)

[...] Mais

un soir ma mère pleure, ensuite je l'ai demandé est-ce qu'elle

pleure et elle ma répondue non je ne pleure pas.

\subsection{Hypothèses}

Ces exemples marquent de façon tout à fait typique à mon sens, la difficulté à $s$ 'approprier le système énonciatif : ce qui peut passer inaperçu à l'oral où la communication s'établit par de multiples canaux, apparaît largement incomplet à l'écrit.

Dans ces cas si communément observés, trois problèmes imbriqués constituent

dro doit faire une rédaction (mise en abyme évidente !) dans laquelle il raconte ce que font ses parents le soir ; en réalité, cette rédaction, dont le sujet est imposé à toutes les écoles de ce pays d'Amérique du Sud, est le système diabolique qu'ont trouvé les militaires de la junte au pouvoir pour espionner leurs concitoyens et surprendre les opposants au régime grâce à l'innocente dénonciation de leurs enfants.

(15) Les élèves (CM2) étaient invités à raconter un épisode de leur vie, bon ou mauvais. 
des caractéristiques d'appropriation " difficile» de la langue par le sujet-scripteur : d'abord, la possibilité pour « je » d'être fictionnel : c'est ce qui se passe quand l'origine de « je » ne coïncide pas avec celle du scripteur ; ensuite, la possibilité que « je » soit utilisé tantôt au plan du « discours ", tantôt à celui de l'« histoire », par exemple quand « je » est narrateur ; et enfin, le changement de référence de « je " embrayeur.

Il me semble que nous voyons ici une illustration des thèses vygotskiennes sur le langage intérieur de l'enfant : la communication écrite ordinaire (dans laquelle «je» et « tu » sont des allocutaires réels) peut être vue comme l'équivalent des premiers échanges sociaux de l'enfant : sur ce modèle, le jeune scripteur commence sans trop de difficultés à dialoguer lui-même par écrit (échange de lettres par exemple comme on en voit fréquemment dans les premiers écrits de CE1). Puis, dans les étapes du développement, vient une période de langage "égocentrique» oral qui est, selon le théoricien, une première forme extériorisée de la pensée, une tentative pour penser, c'est-à-dire pour distinguer sa pensée propre de celle d'autrui. On pourrait voir dans la multiplicité des décrochages énonciatifs dont témoignent les dialogues représentés par les jeunes scripteurs, une analogie avec cette tentative de l'enfant pour penser l'altérité.

Dans l'impossibilité de mettre à distance pensées et paroles d'autrui (pour le scripteur, «autrui », fictif ou non, peut être représenté tantôt comme un « il », tantôt comme un « je »), le scripteur ne peut concevoir le discours d'autrui pris en charge par un « je » qui ne référerait pas à lui, tout comme il ne peut concevoir que ce « je » puisse, lors d'un changement de strate énonciative, devenir un « il ». La représentation des pensées et paroles d'autrui revêtue par un « je » est provisoirement impossible.

Pour Vygotski, le langage «égocentrique » cède lorsque une pensée personnelle parvient à s'intérioriser. Très souvent, l'appropriation de l'incise de discours rapporté - incursion d'un segment narratif dans les paroles rapportées - traduit cette « mise en ordre » intérieure, quand bien même le scripteur n'a pas la maitrise de la forme écrite qui lui correspond : nous voyons en effet avec EX14 et EX15 la forme de l'expression * dije altérer fugitivement la forme du contenu : en effet le lecteur non habitué aura du mal à interpréter dis-je sous cette forme inédite, mais le scripteur ne se trompe pas de niveau narratif:

\section{EX14}

\begin{tabular}{|l|}
\hline 6SYL(2) CJ07 Version 1 \\
\hline $\begin{array}{l}\text { Nous arrivon rapidement a sont antre } \\
\text { Puis le Ciclope commence a nous regarder puis viens nous voir. } \\
\text { personne } \\
\text { Il dis « Dis moi petit homme comment t'appelle tu Je mapelle Hysse } \\
\text { dije, je te defi en comba. Et je pre une lance et luis [plante] dans l'oeye[...] }\end{array}$
\end{tabular}

\section{EX15}

13 CIN(1)CJ07 (version 1)

.....le géant

se retourna je courus or de la grote mais le géant

me ratrapa et me dit tu vouler me tué oui dije

Ainsi, pour donner de l'écriture scolaire une première approche en « négatif ", on pourrait dire qu'elle est caractérisable par une somme d'idiolectes qui manifes- 
tent (donnent à voir) « en creux » la mise en fonctionnement de la langue ; l'écriture manifeste le code et le sujet qui l'investit dans tous ses accidents.

Par ailleurs, le caractère partagé des manifestations « idiolectales » (par rapport à la langue) dans la grammaire des fautes peut être approché d'une autre façon : nous avons dit plus haut que ce paradoxe (à savoir : des formes non normées du code qui fonctionnent collectivement comme une lecte commune aux apprenants ) se résout dès que l'idiolecte est ramené à la norme mais le passage à la norme s'accompagne souvent de la rencontre par l'élève de ce qui est commun dans la langue sous la forme de stéréotypes, d'expressions figées ou semi-figées ; l'appréhension des stéréotypes est alors une autre forme que prend l'écriture scolaire quand elle expérimente le code. Idiolecte et stéréotype seraient ainsi deux façons opposées l'une hypo-normée, l'autre hyper-normée de manifester la langue par l'écriture.

\subsection{La rencontre des stéréotypes}

L'appropriation de la langue par le jeune scripteur s'effectue dans la rencontre des stéréotypes.

Une première définition de ceux-ci concerne la forme de l'expression : tous les linguistes ont remarqué l'existence de formes figées dans la langue, de combinaisons de syntagmes (voire de morphèmes) dont le degré de liberté est moindre pour le locuteur. Ainsi Jakobson :

« Dans toute langue, il existe aussi des groupes de mots codés appelés mots-phrases. La signification de la formule comment ça va ne peut être déduite de l'addition des significations de ses constituants lexicaux; le tout n'est pas égal à la somme de ses parties. Ces groupes de mots, qui sous ce rapport se comportent comme des mots uniques, représentent un cas commun mais néanmoins marginal. Pour comprendre l'écrasante majorité des groupes de mots, il nous suffit d'être familiarisés avec les mots constituants et avec les règles syntaxiques de leurs combinaisons. A l'intérieur de ces limites, nous sommes libres d'ordonner les mots dans des contextes neufs. Bien sûr, cette liberté est relative et la pression des clichés courant sur le choix des combinaisons est considérable ${ }^{(16)}$. Mais la liberté de composer des contextes tout à fait nouveaux est indéniable malgré la probabilité statistique relativement faible de leur occurrence. ${ }^{(17)}$

\subsubsection{Formes figées dans le discours}

On rencontre souvent chez les jeunes scripteurs de ces expressions figées et collocations écrites qui entreraient dans une stylistique de l'expressivité chez Bally ou Frei, comme en témoignent les exemples suivants marqués par le trait « intensif ». Cette grammaire est expressive, comme l'aurait dit Frei en élève de Bally. Par exemple, les formes « fou, folle de joie " "cri de joie », sont fréquemment reprises, mais avec quelques déplacements (cri de joie $\rightarrow$ crier de joie $\rightarrow$ crier très fort) qui les signalent justement comme faits de langue/de style. En voici quelques exemples, au hasard des corpus :

- ma grand-mère était folle de joie. Parce

qu'elle a vu mon ballon très joli et elle a crié très fort (10LAWM2LP05)

(16) Je souligne.

(17) Jakobson, ibid. p. 47. 
et touts le monde criaièrent de joua (17ADR6DB04)

...les applaudissement et les crient de joie des invités (1ELO6DB04)

Ces collocations fonctionnent comme des « signaux », plus en raison de leur excès que de leur banalité, car c'est justement ainsi qu'elles connotent leur appartenance à un « style " scolaire.

\subsubsection{Défigements?}

D'autres « accidents " du mécanisme syntagmatique se retrouvent nombreux dans les stéréotypes de discours :

EX16

\begin{tabular}{|l|}
\hline 2NIC (1) CJ 07, version 3 (réorthographiée) \\
\hline de rep.........'est dur \\
de repre le bateau qui est entouré de \\
Cyclopes curieux. Grâce à notre taille nous pouvons \\
rentrer inaperçus.
\end{tabular}

Dans EX16 il y a tout lieu de penser que c'est la construction «passer inaperçu » qui était visée par le scripteur : ici il s'agit d'une substitution qui « casse » la collocation et dont le caractère accidentel (sporadique) attire l'attention ; mais contrairement aux erreurs idiolectales, cette rencontre peut passer paradoxalement pour une création par défigement, en ce sens que la forme stéréotypée reste à l'arrièreplan de la lecture comme un souvenir qu'on ne reconnaît pas immédiatement.

Pour qu'il ait style scolaire, il faudrait donc alors en passer par la banalité du stéréotype, autre nom de la norme ? Nous allons prendre quelques exemples - positifs cette fois - de rencontres avec la stéréotypie.

\section{Quelques exemples de style comme variété}

Donner une définition du stéréotype en soi n'a d'intérêt que si on le situe dans une problématique. L'intéressant colloque d'Albi qui s'est tenu en 2000 (actes dans Marges Linguistiques, mars $2001^{(18)}$ ) permet de s'orienter dans ce domaine : dans son introduction, V. Fillol situe le stéréotype en relation avec la sémiotique, les théories pragmatique et de l'énonciation. C'est en le définissant comme praxis discursive, que cette approche serait la plus intéressante pour la didactique : le stéréotype y est assimilé à un " lieu commun " au sens propre, un terrain d'entente partagé entre allocutaires qui s'approprient la langue toujours traversée d'une « hétérogénéité constitutive» (J. Authier-Revuz), ce qui accentue sa nature dialogique.

Le stéréotype ou le lieu commun est étudié comme producteur de significations virtuelles, ou encore comme indice de lisibilité des discours, comme stabilisateur de sens, ce qui permet l'appropriation des genres. C'est une approche différente de la vision négative et pessimiste de Barthes évoquant la paralysie de l'énonciation, dans l'impossibilité à énoncer autre chose que du déjà dit.

Ces aspects contradictoires coexistent d'ailleurs.

(18) Colloque d'Albi, Langages et signification. 
L'approche barthésienne met l'accent plus particulièrement sur le dépôt culturel représenté par les formes comme sédimentées de lieux communs mais c'est aussitôt pour se demander comment de telles formes ("patterns ») se transforment en « style» et sous quel effet :

« ...je voudrais simplement tirer une hypothèse de travail : considérer les traits stylistiques comme des transformations, dérivées soit de formules collectives (d'origine irrepérable, tantôt littéraire, tantôt pré-littéraire ), soit, par jeu métaphorique, de formes idiolectales ; dans les deux cas, ce qui devrait dominer le travail stylistique, c'est la recherche de modèles, de patterns: structures phrastiques, clichés syntagmatiques, départs et clausules de phrases ; et ce qui devrait l'animer, c'est la conviction que le style est essentiellement un procédé citationnel, un corps de traces, une mémoire [...], un héritage fondé en culture et non en expressivité. Ceci permet de situer la transformation à laquelle on fait allusion [...] : elle peut avoir quelques affinités avec la grammaire transformationnelle, mais elle en diffère sur un point fondamental $[\ldots]$ : les « modèles » stylistiques ne peuvent être assimilés à des «structures profondes ", à des formes universelles issues d'une logique psychologique ; ces modèles sont seulement des modèles de culture (même s'ils semblent très anciens) ; ce sont des répétitions, non des fondements ; des citations, non des expressions ; des stéréotypes, non des archétypes. " Barthes, O.C., T. III, p .980, « Le style et son image », colloque de Bellaggio, 1969, [Literary Style : a Symposium », Seymour Chatman, Oxford University Press, 1971.]

En didactique, on pourrait faire l'hypothèse que le style est un passage de l'idiolecte négatif vers le stéréotype considéré comme l'accès au bien commun partagé ${ }^{(19)}$ et retour : la variation du stéréotype recrée alors des styles d'écriture scolaire. On retient donc le stéréotype comme praxis discursive, en relation avec les genres scolaires du récit ou de la description.

J'ajoute que, dans la description sémiotico-rhétorique qu'en donne Dufays pour la lecture, la stéréotypie est toujours le fait du lecteur, qui interprète ce qu'il lit à travers ses connaissances intertextuelles et son univers de valeurs. On ne donnera donc pas à la stéréotypie le sens péjoratif qu'elle a d'habitude dans la mesure où elle est conçue comme un moyen d'enseignement des codes de lecture et d'écriture, codes indispensables à tout apprentissage.

Dans les exemples suivants, deux types de stéréotypie vont être examinés : une stéréotypie relevant de la forme du contenu : topoï et motifs narratifs, scripts, scénarios, structure énonciative et narrative dans l'écriture de l'épisode d'un récit; et une stéréotypie relevant de la forme de l'expression : figures, syntagmes et expressions idiomatiques. Dans les deux cas, ces stéréotypies relèvent du genre scolaire du récit.

\subsection{La stéréotypie comme appropriation des codes rhétoriques}

Voici un premier exemple de copie qui assimile le code scolaire tant dans la forme du contenu (lois du genre) que dans la forme de l'expression (syntagmes), manifestant ainsi son aptitude à réécrire et imiter.

(19) Je reprends ici une remarque très suggestive de F. Rastier citant les écrits pédagogiques de Schleiermacher et relevant qu'aux yeux du philosophe allemand, apprendre consiste pour l'enfant à abandonner les genres idiosyncrasiques et les inventions linguistiques "pour s'intégrer dans la vie de la langue commune » (Pincemin, B. et Rastier, F. 1999 : 111). 


\begin{tabular}{|l|}
\multicolumn{1}{c|}{ Le déménagement } \\
Si vaR(M2)LP05 ${ }^{(20)}$ (transeription du texte original) \\
je vais vous parler de mon déménagement. \\
J'habitais dans un très beau quartier, ou il fait toujours beau, mais si il \\
le fait trop, on pouvait toujours trouver une place à l'ombre. \\
A l'école, la récréation durait au moins 30 min et les cours \\
environ 2h30. Dès que l'on arrivait à l'école, on entrait directement \\
en classe, pour ne pas faire perdre du temps à la classe. Mon meilleur \\
copain, Valentin B***, je le connnaîssait depuis la petite section et \\
je jouais toujours avec lui à la récréation. On s'amusait \\
comme des fous et au CP,CEl on était fiers de nous, de notre \\
travail. Jusqu'au jour où...tout bascula subitement. Le \\
29 Juin, à la fin du CE1, ma mère et mon père m'apprirent \\
la plus mauvaise nouvelle de ma vie, qui allait \\
tout boulverser... nous allions... déménager. Je pouvais dire au \\
revoir au beau quartier rayonnant, au revoir à l'école \\
de rêve, au revoir à Valentin... Alors là je me \\
suis mis à crier à hurler et dire que si on déménageait, \\
je quitterai cette maison et on me regretterait beaucoup... \\
mais malheureusement, cela n'a pas marché. 3 mois plus tard, \\
on a déménagé et moi, dans la voiture, je restait \\
dans mon coin et toute ma vie défiler devant moi... \\
J'était fíchu !
\end{tabular}

On peut tout à fait interpréter ce récit en référence à un « lieu » rhétorique, 1'invention consistant dans le changement «possible " ${ }^{(21)}$ mais brutal d'une situation ( $c f$. 1. 11, exactement au milieu du récit) et surtout, dans la portée axiologique qui lui est attribuée. Ce sont les contraires que le scripteur met en relation qui donnent sens à l'événement : la dimension de contraste temporel (non accompli vs accompli), le parcours sémiotique qui conduit du contenu inversé au contenu posé ${ }^{(22)}$, le tout en vingt lignes, attestent chez l'élève la connaissance du genre de l'" anecdote ". On admire au passage l'humour des lignes 3-4 magistralement reprises ligne 15 en une synthèse évoquant le "beau quartier rayonnant", la distance humoristique (lignes 16-19) mais aussi l'émotion des deux dernières lignes (21-22) reprenant l'avertissement de la première ligne, etc.

La singularité de la mise en mots repose pourtant sur l'emploi de nombreux stéréotypes lexicaux ou de collocations: $s$ 'amuser comme des fous, l'école de rêve, la plus mauvaise nouvelle de ma vie, toute ma vie défilait devant moi... Mais ces stéréotypes (ou ces collocations) sont pris(e)s dans la mécanique du renversement qui caractérise ce récit : c'est la forme du contenu (le topos) qui est prégnante, elle se projette sur la forme de l'expression et « aveugle » la lecture des stéréotypes.

Inversement, l'attention est attirée sur un exemple particulier de forme de l'expression : dire au revoir (lignes 14 à 16) vs dire adieu. Au lieu de : "dire adieu ",

(20) La consigne était la suivante pour cette classe d'élèves de CM2 de la banlieue nord parisienne: "Vous allez écrire une petite autobiographie. Vous devrez raconter un bon ou un mauvais souvenir de votre vie ( 15 à 20 lignes) ».

(21) Lieu du possible et de l'impossible chez Aristote, par exemple.

(22) Par exemple A. J. Greimas, Du Sens, Paris : Seuil, 187-188 et $s q$. 
stéréotype lexical, dont le sens « considérer comme perdu, renoncer à » serait attendu pour dramatiser l'événement raconté, l'élève utilise le syntagme courant : " dire au revoir ", apparemment synonyme, dont le sens est limité à : "prendre congé de »; mais en même temps, il souligne cet emploi par un triplement (lignes 14-16).

Innovation ou maladresse, approximation voulue ou fait de style? Il me semble que cet exemple illustre la grande difficulté pour le lecteur de textes d'élèves, à déterminer le passage qui mène de l'idiolecte, qu'on pourrait définir comme une méconnaissance créative de la norme, au stéréotype comme appropriation du code commun, et retour. En effet, si l'accès à la langue se fait par un lent abandon de l'idiolecte "négatif ", tel qu'il a été défini précédemment, c'est qu'il se produit - en même temps - la rencontre du stéréotype dans sa banalité de discours commun. Plus que d'une question d'ordre dans le temps, c'est à la reprise/ modification (F. François) du stéréotype, que se mesure l'entrée dans ce que je nomme un «style d'écriture scolaire ".

\subsection{Stéréotypie et re-création d'idiolectes}

\section{Le deuxième exemple prend appui sur un corpus.}

Je propose une série de variations observées dans un corpus de copies d'élèves de $6^{\mathrm{e}}$ qui ont pour consigne d'imaginer une scène de mariage entre mots, en l'occurrence des adjectifs et des noms. Le texte à produire s'écrit à partir du roman d'E. Orsenna «La grammaire est une chanson douce » ${ }^{(23)}$, fable didactique dans laquelle les mots sont des personnages doués de vie. Dans le passage à inventer, la scène repose sur l'utilisation métaphorique du lexème " accorder » qui, sans perdre son sens métalinguistique grammatical, lui superpose - du fait de la personnification des mots - un sens matrimonial.

Deux stéréotypes sont à l'œuvre dans cette scène :

- un stéréotype de contenu, qui est le topos du scénario du consentement entre époux, marqué par la présence du maire et des deux époux. Ce stéréotype correspond au script «échange des consentements » et comporte un dialogue stéréotypé. Mais la répétition du stéréotype n'est pas pour autant identique dans les copies ;

- un stéréotype de forme, marqué par les formules ritualisées du dialogue direct, qui manifeste l'échange de consentements. Bien entendu, les « meilleures copies » sont celles qui parviennent à maintenir l'ambivalence sémantique et lexicale dans l'accord des mots.

La présence d'un modèle sous-jacent tant dans la forme que dans le contenu (à savoir, les deux stéréotypes qui viennent d'être mentionnés), nous permet de cerner ce qui peut être dit « style » dans les copies. Ce sont en effet les variantes observées dans la reproduction des deux stéréotypes, c'est-à-dire les « transformations » issues des " modèles » barthésiens (voir supra) que j'appellerai donc "style ».

On peut en recenser de deux sortes :

- les variantes affectant le script sous sa forme dialoguée,

- les variantes affectant le double sens de « accorder"

(23) Orsenna, E. (2001) : La grammaire est une chanson douce, Paris : Stock. 
Les exemples EX18 à EX20 illustrent diverses formes de compréhension du script « mariage » appliqué au mariage des mots :

EX18

\begin{tabular}{|l|}
\hline 7RIW6DB04 V3 \\
Lye maire voyant que « maison » et « hanté » étaient \\
préssés, il commença tout de suite le discours : \\
- « Hanté », voulez-vous prendre pour nom « Maison »? \\
- Oui ! répondit « hanté » d'un air impatient. \\
[...] \\
Le maire recommença le discours avec « maison »: \\
- « Maison », voulez-vous prendre pour adjectif « hanté "?
\end{tabular}

\section{EX19}

\begin{tabular}{|c|c|}
\hline $12 E$ & LOG6DB04 V2 (1) \\
\hline 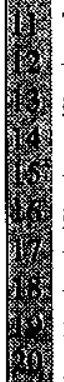 & $\begin{array}{l}\text { Tout à coup le maire arriva. Il disa : } \\
\text { - "Hanté » voulez-vous prendre pour } \\
\text { accordement « Maison »? « Hanté » repondit : } \\
\text { - oui je le veux. } \\
\text { - « Maison " voulez-vous prendre pour } \\
\text { accordement « Hanté " ? Maison repondit : } \\
\text { - oui je le veux. Avec grand plaisir. Le maire dit à son tour : } \\
\text { - « Maison » et « Hanté » vous vôla } \\
\text { mari et femme et vous voîla aussi « Maison Hanté ». Vous pouvez embrassé } \\
\text { la marier. }\end{array}$ \\
\hline
\end{tabular}

EX20

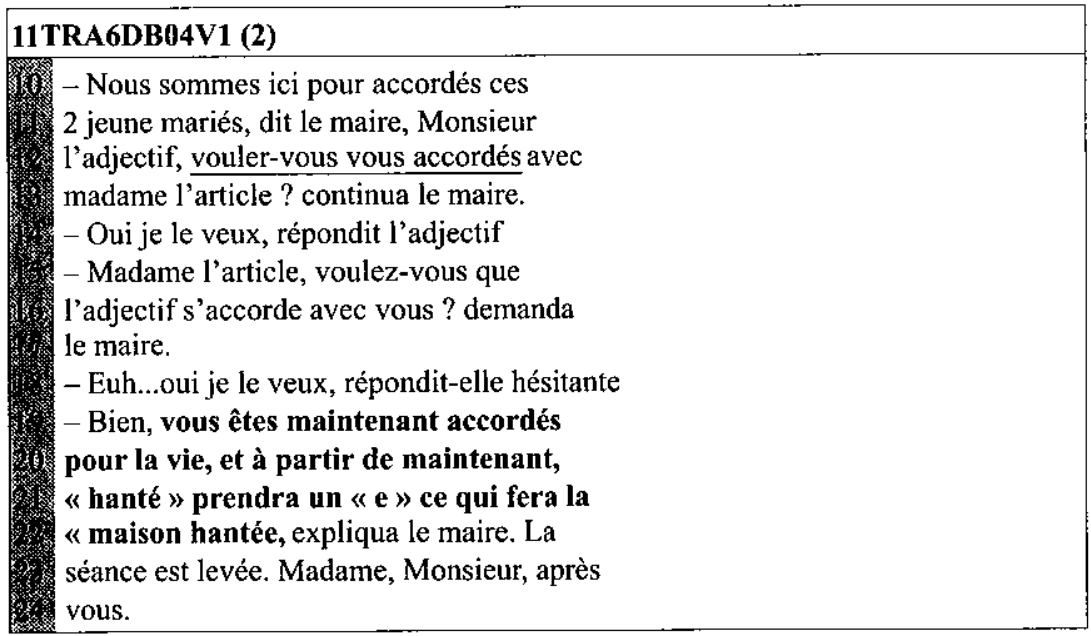

On note les formes de différenciation du stéréotype : elles vont de la création de néologismes (prendre pour accordement) à la transposition du syntagme figé " prendre pour mari/femme » en " prendre pour adjectif/nom » ou encore le discours d'adresse à l'adjectif et l'article pour s'accorder, la fiction grammairienne ne reculant devant rien! (EX20, lignes $19 s q$.).

Dans ce cas, le dialogue fonctionne par substitution des formules ritualisées mais de sorte que la structure de la phrase reste évidemment compréhensible : 
c'est par le détournement du modèle stéréotypé qu'on peut dire que celui-ci est assimilé.

Le tableau suivant (EX21) note le cheminement d'un autre élève vers l'appropriation de la norme.

\section{EX21}

\begin{tabular}{|l|c|c|}
\hline 18PIV6DB04 & \\
(transcription du texte dans la présentation et l'orthographe d'origine)
\end{tabular}

La lecture des changements intervenus dans les trois versions montre en effet une compréhension progressive vers le stéréotype attendu, à savoir : le maintien de l'ambivalence lexicale du verbe " accorder ", d'une part, et d'autre part, la personnification des noms. La substitution des formes "prendre comme homme " (version 1, 1. 15) vs " prendre comme mari" (version 2 ligne 14) vs « s'accorder avec " (version 3 ligne 13) est bien en ce sens un passage de l'idiolecte vers le stéréotype. De même, l'équivoque entre le substantif " mari » et le verbe " marie " dans l'expression idiolectale « je vous marie et femme » (versions 1 et 2 , dernière ligne) retrouve la norme dans la dernière version : pour cet élève, c'est le passage de l'idiolecte « pittoresque» (mais hors de la norme) vers le stéréotype de la forme « accorder » condensant le double sens requis par le contexte, qui constitue la réponse stylistique scolaire adéquate; le «style scolaire » retrouve bien ici ses caractéristiques de conformité à la norme scolaire (linguistique, textuelle, générique, discursive).

Cependant, si ce qui fait « style » c'est, comme l'a bien vu Barthes (cf. supra), la "transformation des modèles, des patterns », les moyens de cette transformation sont évidemment imprévisibles : il n'existe pas de " grammaire transformationnelle " du style.

Par exemple, la copie ci-dessous (EX22) est toute colorée de sentimentalisme : ce qui retient l'attention, dans la version 1, c'est l'impatience (du narrateur ? ou des personnages ?) manifestée au discours du maire qualifié de « discours barbant ", et son résumé irrévérencieux (tatati et tata), le baiser des amoureux, le 
choix du verbe «chérir » dans la formule de recommandation du maire aux époux; la phrase finale de la version 2 (peu conforme aux formules du dialogue stéréotypé) prime sur les contraintes de la consigne et finalement dans la version 3 , les mots autonymisés et personnifiés ne se distinguent plus des humains : ils sont déclarés «mari » et « femme » parce qu'ils s'aiment :

EX22

\begin{tabular}{|c|c|c|}
\hline \multicolumn{3}{|c|}{$\begin{array}{l}\text { 24LAEV6DB04 } \\
\text { (transcription du texte dans la présentation et l'orthographe d'origine) }\end{array}$} \\
\hline Version 1 (2) & Version 2 (1) & Version 3 \\
\hline \begin{tabular}{|l|} 
Puis vint le moment du \\
discours barbant. \\
- Vous l'aimez, vous \\
la chérirait tatati et \\
tata \\
Et la question finale : \\
- Hanté, voulez-vous \\
prendre pour épousse \\
Maison? \\
- Oui je le veux \\
- Et vous Maison vou- \\
lais vous pour époux \\
Hanté ? \\
- Oui \\
Ils s'embrassèrent et se \\
tenant toujours par la \\
main, \\
retongnit une musique \\
des pétales de roses vo- \\
L lè- \\
-rent dans tous les sen- \\
ses et sous les Houras \\
des mots
\end{tabular} & $\begin{array}{l}\text { Puis vint le moment du } \\
\text { discours barbant. } \\
\text { - Vous vous aimez, } \\
\text { vous vous chérirait } \\
\text { Et la phrase finale : } \\
\text { - vous aimez-vous? }\end{array}$ & $\begin{array}{l}\text { Puis vint le moment du } \\
\text { discours barbant. } \\
\text { - Vous vous aimez? } \\
\text { ect... ect... } \\
\text { Et la phrase finale : } \\
\text { - je vous déclare marie } \\
\text { et femme }\end{array}$ \\
\hline
\end{tabular}

Qu'est-ce qui fait style dans cet exemple ? Le passage d'une formulation stéréotypée à une autre (version 3) n'efface pas pour le lecteur la nouveauté du « discours barbant». C'est ici de «mélange " (Frédéric François) qu'il faudrait parler.

C'est aussi parfois un détail, comme l'adresse «mes enfants " du maire à l'assistance $(\mathrm{EX} 23,1.28)$ qui suffit à faire varier le stéréotype du dialogue :

EX23

\begin{tabular}{|l|}
\hline 16JUS6DB04 \\
(transcription du texte dans la présentation et l'orthographe d'origine) \\
Soudain le mot maire arriva. \\
- Mes enfants, nous sommes reuni aujourd'hui \\
pour celebrer le mariage du mot maison et de \\
l'adjectif hanté. \\
Je vais donc vous demander de vous levez et de les \\
applaudirent bien fort.
\end{tabular}

D'autres manières de varier existent, elles relèvent des diverses formes de mo- 
tivation qu'ont entraînées la figure de personnification et le double sens d' " accorder » réclamés par la consigne d'écriture : rappelons que les mots " maison » et " hanté » doivent s'accorder à la mairie des mots.

En voici quelques exemples :

- par implication et présupposé : la demoiselle d'honneur est plus «petite» que la mariée «maison» :

\section{EX24}

\begin{tabular}{|l|}
\hline 14YOH6DB04Version 2 Etat 1 \\
W.la \\
demoiselle d'honneur, le nom « maisonnette » \\
trépignait d'impatience \\
\hline
\end{tabular}

— par mimétisme (analogie) du référent :

\section{EX25}

\begin{tabular}{|l|}
\hline 24LAEV6DB04 Version 2, Etat \\
La maison portait une robe toute blanche et \\
sur ses cheveux rouge vif elle portait un magnifique chapeau \\
[...] \\
Hanté n'avait pas beaucoup changeait, toujours au \\
ssi blanc il n'avait qu'un élégant nœud, papillion noir.
\end{tabular}

- par mimétisme (analogie) du signifié :

\section{EX26}

\section{MAR6DB04 Version 2, Etat 2}

- Je suis contente que

vous soyez venue ! Dit « maison » au mot joie

- Mais c'est un plaisir ! répondit-elle.

— par métalepse :

EX27

\begin{tabular}{|l|}
\hline 16JUS6DB04 COPIE Version 3 \\
$\begin{array}{l}\text { Le nom voyage était une personne très } \\
\text { cultivée elle était noir de peau car elle venait d'un } \\
\text { pays lointain d'Afrique. }\end{array}$ \\
\hline
\end{tabular}

Ce qui ressort de ces variations est bien le caractère imprévisible des détails de la transposition tant dans le contenu que dans l'expression.

Le style serait alors dans l'imprévisibilité paradoxale du stéréotype, sa recréation indéfinie dans la mise en mots, marque ultime de l'appropriation de la langue par le sujet. 


\section{Conclusion}

Au terme de ce parcours, je retiendrai le caractère dynamique et continu de l'appropriation de la langue par le sujet scolaire : l'écriture scolaire est en effet le passage d'idiolectes d'apprenants - relativement prévisibles si l'on considère le système de la langue - à la créativité, elle aussi dans la langue mais imprévisible, permise par la rencontre et le dépassement des stéréotypes. Ainsi deux traits me semblent relever d'un "style» dans le domaine de l'écriture scolaire : la variété des passages de l'idiolecte «négatif » (au regard de la norme) au stéréotype, et le caractère imprévisible de la «transformation » de structures stéréotypées.

La notion de «passage » est elle-même à approfondir : elle ne désigne pas le trajet d'un apprentissage, puisque tout un chacun passe sans cesse de l'idiolecte au stéréotype et retour, mais le mouvement de l'activité langagière elle-même.

Par ailleurs, les notions d'idiolecte et de stéréotype, comme celles de style et écriture, ont été prises à différents niveaux de structuration des unités de la langue, tant d'expression que de contenu; or ces niveaux dépendent eux-mêmes de contextes et de genres, ce qui signifie que le scripteur scolaire, s'il s'individualise comme sujet par l'acte d'écrire, partage avec d'autres les traits globaux d'une écriture scolaire.

\section{Références citées}

BARTHES, R. (1953 et 1964): Le degré zéro de l'écriture, suivi de Eléments de sémiologie Paris, Le Seuil, Gonthier.

- (2002, édition E. Marty) : Euvres complètes, Tomes III, IV et V, Paris, Le Seuil.

DuFAYS, J.-L. (1994) : Stéréotype et lecture, Liège, Mardaga.

FILLOL, V. (2001) : «Le stéréotype comme cliché-concept et comme concept-clé en analyse du discours ", in Marges linguistiques publication en ligne archive texto.net, mars 2001, pp. 1-17

HJELMSLEV, L. : ([1943]1968-1971 $2^{\mathrm{e}}$ trad.) : Prolégomènes à une théorie du langage, Paris, Minuit.

JAKOBSON, R. (1963) : Essais de linguistique générale, I, chapitre IX, « Les embrayeurs, les catégories verbales et le verbe russe ", Paris, Minuit.

KYHENG, R. (2005) : «Hjelmslev et le concept de texte en linguistique ", in Texto, septembre 2005, vol X, n ${ }^{\circ} 3$.

FRANÇOIS, F. (2006) : Rêves, récits de rêves et autres textes, Limoges, Lambert-Lucas.

FREl, H. ([1929], 1993) : La grammaire des fautes, Slatkine Reprints. 